\title{
Inferences for generalized Topp-Leone distribution under dual generalized order statistics with applications to Engineering and COVID-19 data
}

\author{
Devendra Kumar ${ }^{\mathrm{a}, *}$, Mazen Nassar ${ }^{\mathrm{b}, \mathrm{c}}$, Sanku Dey ${ }^{\mathrm{d}}$ and Ahmed Elshahhat ${ }^{\mathrm{e}}$ \\ ${ }^{a}$ Department of Statistics, Central University of Haryana, Haryana, India \\ ${ }^{\mathrm{b}}$ Department of Statistics, Faculty of Science, King Abdulaziz University, Jeddah, Kingdom of Saudia Arabia \\ ${ }^{\mathrm{c}}$ Department of Statistics, Faculty of Commerce, Zagazig University, Zagazig, Egypt \\ ${ }^{\mathrm{d}}$ Department of Statistics, St. Anthony's College, Shillong, Meghalaya, India \\ ${ }^{\mathrm{e}}$ Faculty of Technology and Development, Zagazig University, Zagazig, Egypt
}

\begin{abstract}
This article accentuates the estimation of a two-parameter generalized Topp-Leone distribution using dual generalized order statistics (dgos). In the part of estimation, we obtain maximum likelihood (ML) estimates and approximate confidence intervals of the model parameters using $d g o s$, in particular, based on order statistics and lower record values. The Bayes estimate is derived with respect to a squared error loss function using gamma priors. The highest posterior density credible interval is computed based on the MH algorithm. Furthermore, the explicit expressions for single and product moments of dgos from this distribution are also derived. Based on order statistics and lower records, a simulation study is carried out to check the efficiency of these estimators. Two real life data sets, one is for order statistics and another is for lower record values have been analyzed to demonstrate how the proposed methods may work in practice.
\end{abstract}

Keywords: Generalized Topp-Leone distribution, order statistics, dual generalized order statistics, lower record values

\section{Introduction}

Shekhawat and Sharma (2020) introduced a new extension of Topp-Leone (TL) distribution on the unit interval by adding a skewness parameter in the TL distribution using the power transformation called the generalized Topp-Leone (GTL) distribution. The probability density Eq. (1) of GTL distribution is given by

$$
f(y ; \tau, \delta)=2 \tau \delta y^{\tau \delta-1}\left(1-y^{\delta}\right)\left(2-y^{\delta}\right)^{\tau-1}, 0<y<1, \tau, \delta>0,
$$

and the corresponding cumulative distribution Eq. (2) and hazard function are given by

$$
F(y ; \tau, \delta)=\left\{y^{\delta}\left(2-y^{\delta}\right)\right\}^{\tau}, 0<y<1, \tau, \delta>0
$$

It can be seen that

$$
\left(2 y-y^{\delta+1}\right) f(y)=2 \tau \delta\left(1-y^{\delta}\right) F(y)
$$

\footnotetext{
${ }^{*}$ Corresponding author: Devendra Kumar, Department of Statistics, Central University of Haryana, India. E-mail: devendrastats@ gmail.com.
} 
Topp-Lone distribution is a member of GTL distribution if $\delta=1$. Hazard function of GTL is increasing and bathtub shaped. For more details and application of this distribution, readers may refer to Shekhawat and Sharma (2020).

Bounded distributions are increasingly gaining grounds in literature owing to their significance in several areas like psychology, economics, biology, engineering and many others. For instance, in psychology, proportions and percentages play a vital role in evaluating the probability of judgments (Smithson \& Shou, 2017). Similarly in economics, one may come across several instances where data are bound on the unit interval. For example, proportion of income spent on non-durable consumption, pension plan participation rates, market shares, fractional repayment on debts and capital structures (Ghosh et al., 2019; Papke \& Wooldridge, 1996; Smithson \& Shou, 2017). Besides, for measuring reliability, it is imperative to have models defined on the unit interval in order to have plausible results (Genç, 2013).

Distributions based on unit interval are known to have desirable failure (hazard) rate characteristics such as increasing, decreasing and bathtub shapes. However, one may encounter situations where only increasing and bathtub failure rates are used or observed. These failure rate characteristics are vital when modeling datasets. For instance, Rajarshi and Rajarshi (1998), and Lawless (2003) in their studies observed that distributions with bathtub hazard rates are needed to model lifetime of electronic, electrochemical and mechanical products; while Lai (2013) observed that the optimum number of minimal repairs for systems have increasing failure rates. Also, it has been observed that during clinical development drugs have increasing failure rate (see Woosley and Cossman (2007)).

The concept of generalized order statistics (gos) has held the attention of statisticians for a long time. It was first proposed by Kamps (1995) and includes ordered random variables arranged in increasing order of magnitude such as order statistics, sequential order statistics, progressive type II censored order statistics, records and Pfeifers records. However, the ordered random variables which are arranged in decreasing order of magnitude can not be studied in this framework. Owing to this, statisticians felt the need for the ordered random variables which can also be arranged in decreasing order of magnitude. For example, the life length of an electric bulb arranged from highest to lowest. The study of distributional properties of such random variables by using the inverse image of gos is popularly known as dual generalized order statistics. Pawlas and Szynal (2001) first proposed the concept of dual generalized order statistics (dgos) wherein the order random variables can be studied in both increasing and decreasing order of magnitude. This concept was further studied in a systematic manner by Burkschat et al. (2003). Dual gos includes order statistics (reversed ordered order statistics), lower k-records and lower Pfeifer records. For better understanding, dgos can be used when $F(y)$ is in compact and closed form and while gos can be used when $1-F(y)$ is in closed and compact form.

In the last two decades or so, several studies have been carried out on the statistical properties of continuous distributions based on dgos. In this regard, readers may refer to the works of Pawlas and Szynal (2001), Ahsanullah (2004, 2005), Mbah and Ahsanullah (2007), Anwar and Athar (2008), Barakat and El-Adll (2009), Khan et al. (2010), Khan and Kumar (2010, 2011), Jaheen and Al Harbi (2011), Athar and Faizan (2011), Kumar (2013a, b), Khan and Khan (2015), Kumar (2016), Li (2016), Kumar and Dey (2017), Khan and Iqrar (2019), Kumar et al. (2020) among others.

However, to the best of our knowledge, there are no reports on GTL distribution based on dgos. The motivation of the paper is two fold: first is to derive the explicit expressions for the single and the product moments based on dgos of GTL distribution. Second is to estimate the parameters of the model from both frequentist and Bayesian view points based on order statistics and lower record values.

The paper is organized as follows. In Section 2, we present preliminaries of dgos. In Section 3, we present explicit expressions for the single moments and product moments of order statistics of $d g$ os of GTL distribution. In the same Section, we reported the mean and variances of order statistics and lower record values. Two methods of estimation namely, maximum likelihood method of estimation and Bayesian method of estimation are discussed in Section 4. To obtain the Bayes estimates, independent gamma priors of the unknown model parameters are used under squared error loss (SEL) function. In Section 5, simluation study is carried out to evaluate the performance of the ML and the Bayes estimates based on root mean squared error (RMSE) and relative absolute bias (RAB). In addition, average length (AL) and coverage percentages (CPs) for the 95\% approximate confidence interval (ACI) and highest posterior density (HPD) credible intervals of the parameters under order statistics and lower record values is provided in the same Section. We illustrate the methodology developed in this manuscript and the usefulness of the GTL distribution 
based on two real-life data sets, one is for order statistics and another is for lower record values in Section 6. Finally, concluding remarks are provided in Section 7.

Some lemmas useful for the derivation of the explicit expressions are provided in APPENDIX. The lemmas make use of the Gauss hypergeometric function and Kampé de Feriet's Function defined by

$$
{ }_{2} F_{1}\left(b_{1}, b_{2}, b_{3} ; y\right)=\sum_{k=0}^{\infty} \frac{\left(b_{1}\right)_{k}\left(b_{2}\right)_{k} y^{k}}{\left(b_{3}\right)_{k} k !}
$$

and

$$
F_{l: m ; n}^{p: q ; k}\left(\begin{array}{c}
\left(a_{p}\right):\left(b_{q}\right) ;\left(c_{k}\right) \\
\left(\alpha_{l}\right):\left(\beta_{m}\right) ;\left(\gamma_{n}\right)
\end{array} x, y\right)=\sum_{r=0}^{\infty} \sum_{r=0}^{\infty} \frac{\prod_{j=1}^{p}\left(a_{j}\right)_{r+s} \prod_{j=1}^{q}\left(b_{j}\right)_{r} \prod_{j=1}^{k}\left(c_{j}\right)_{s}}{\prod_{j=1}^{p}\left(\alpha_{j}\right)_{r+s} \prod_{j=1}^{q}\left(\beta_{j}\right)_{r} \prod_{j=1}^{k}\left(\gamma_{j}\right)_{s}} \frac{x^{r}}{r !} \frac{y^{s}}{s !},
$$

where $(f)_{p}=f(f+1) \ldots(f+p-1)$ denotes the ascending factorial. In-built routines for computing these special functions are available in packages like Maple, Matlab and Mathematica. For example, Hypergeometric2F1 $\left[b_{1}, b_{2}, b_{3} ; y\right]$ in Mathematica computes the Gauss hypergeometric function.

\section{Dual generalized order statistics and preliminaries}

The random variables $Y(1, n, m, k), Y(2, n, m, k), \ldots, Y(n, n, m, k)$ are said to be dual generalized order statistics (dgos) based on the cdf $F$ if their joint pdf is

$$
f_{Y(1, n, m, k), Y(2, n, m, k), \ldots, Y(n, n, m, k)}\left(y_{1}, y_{2}, \ldots, y_{n}\right)=k \prod_{j=1}^{n-1} \gamma_{j} \prod_{i=1}^{n-1}\left\{F_{i}^{m}\left(y_{i}\right) f\left(y_{i}\right)\right\} F^{k-1}\left(y_{n}\right) f\left(y_{n}\right)
$$

for $y_{1}>y_{2}>\ldots>y_{n}$, where $n \geqslant 2, k>0$ and $-\infty<m<\infty$ are such that $\gamma_{r}=k+(m+1)(n-r)>0$ for all $r=1,2, \ldots, n-1$. It follows that the pdf of the $r$ th $d g o s$ is

$$
f_{Y(r, n, m, k)}(y)=\frac{C_{r-1}}{(r-1) !} F^{\gamma_{r}-1}(y) g_{m}^{r-1}(F(y)) f(y)
$$

for $y>0$, where

$$
C_{r-1}=\prod_{j=1}^{r} \gamma_{j}, \quad g_{m}(y)=\frac{1}{m+1}\left[1-y^{m+1}\right] .
$$

It follows also that the joint pdf of the $r$ th and $s$ th $d g o s$ is

$$
\begin{aligned}
f_{Y(r, n, m, k), Y(s, n, m, k)}(y, z)= & \frac{C_{s-1}}{(r-1) !(s-r-1) !} F^{m}(y) F^{\gamma_{s}-1}(z) g_{m}^{r-1}(F(y)) \\
& \times\left[g_{m}(F(z))-g_{m}(F(y))\right]^{s-r-1} f(y) f(z),
\end{aligned}
$$

for $0<y<z$.

\section{Relations for moments of the dgos from GTL distribution}

In this section, we derive explicit expressions and recurrence relations for single and product moments of $d g o s$ given a random sample $Y_{1}, Y_{2}, \ldots, Y_{n}$ from the GTL distribution. 


\subsection{Relations for single moments of dgos}

Here, first we present the explicit expressions and recurrence relations for rth $d g o s, Y(r, n, m, k)$ of GTL distribution

Theorem 1. For $1 \leqslant r \leqslant n$ and $p=1,2, \ldots$ and let $Y_{1}, Y_{2}, \ldots, Y_{n}$ be the independent and identically distributed random variables of size $n$ from GTL distribution, then the explicit expressions for single moments of pth $d g o s$, $E\left[Y(r, n, m, k)^{(p)}\right]=\alpha_{r, n, m, k}^{(p)}$ is given by

$$
\begin{aligned}
\alpha_{r, n, m, k}^{(p)}= & \frac{2 \delta \tau C_{r-1}}{(r-1) !(m+1)^{r-1}} \sum_{i=0}^{r-1}(-1)^{i}\left(\begin{array}{c}
r-1 \\
i
\end{array}\right) \\
& \times\left\{\Delta_{1}\left(p+\delta \tau \gamma_{r-i}, \tau \gamma_{r-i}\right)-\Delta_{1}\left(p+\delta \tau \gamma_{r-i}+\delta, \tau \gamma_{r-i}\right)\right\}
\end{aligned}
$$

where $\Delta_{1}\left(p_{1}, p_{2}\right)$ is defined in Eq. (24).

Proof From Eq. (4), we get

$$
\begin{aligned}
\alpha_{r, n, m, k}^{(p)}= & E\left[Y(r, n, m, k)^{(p)}\right]=\frac{C_{r-1}}{(r-1) !} \int_{0}^{1} y^{p} F^{\gamma_{r}-1}(y) g_{m}^{r-1}(F(y)) f(y) \mathrm{d} y \\
= & \frac{C_{r-1}}{(r-1) !(m+1)^{r-1}} \sum_{i=0}^{r-1}(-1)^{i}\left(\begin{array}{c}
r-1 \\
i
\end{array}\right) \int_{0}^{1} y^{p}[F(y)]^{\gamma_{r-i}-1} f(y) \mathrm{d} y \\
= & \frac{C_{r-1}}{(r-1) !(m+1)^{r-1}} \sum_{i=0}^{r-1}(-1)^{i}\left(\begin{array}{c}
r-1 \\
i
\end{array}\right) 2 \delta \tau \\
& \times\left\{\int_{0}^{1} y^{p+\delta \tau \gamma_{r-i}-1}\left(2-y^{\delta}\right)^{\tau \gamma_{r-i}-1} \mathrm{~d} y-\int_{0}^{1} y^{p+\delta\left(\tau \gamma_{r-i}+1\right)-1}\left(2-y^{\delta}\right)^{\tau \gamma_{r-i}-1} \mathrm{~d} y\right\} .
\end{aligned}
$$

The result follows by using Lemma 1 . The proof is complete.

Corollary 1. For $m=-1$ in Eq. (6), the explicit expression for the single moments of lower $k$ record values for the GTL distribution can be obtained by using L'Hospital's Rule as

$$
\alpha_{r, n,-1, k}^{(p)}=\sum_{j=0}^{\infty} \frac{2^{-\frac{p}{\delta}-2 j}\left(\frac{p}{\delta}\right)}{j\left(\frac{p}{\delta}+j\right)} \operatorname{Bet}\left(\frac{p}{\delta}+j, j\right)\left(\frac{k \tau \delta}{p+\tau \delta k+\delta j}\right)^{r},
$$

where $\operatorname{Bet}(a, b)$ is the beta function.

Remark 1. Setting $m=0, k=1$ in Eq. (6), the exact explicit formula for single moments of order statistics can be obtained as

$$
\begin{aligned}
\alpha_{n-r+1: n}^{(p)}= & \frac{2 \delta \tau n !}{(r-1) !(n-r) !} \sum_{i=0}^{r-1}(-1)^{i}\left(\begin{array}{c}
r-1 \\
i
\end{array}\right)\left[\Delta_{1}(p+\delta \tau(n-r+i+1), \tau(n-r+i+1))\right. \\
& \left.-\Delta_{1}(p+\delta \tau(n-r+i+1)+\delta, \tau(n-r+i+1))\right] .
\end{aligned}
$$

That is

$$
\begin{aligned}
\alpha_{r: n}^{(p)}= & \frac{2 \delta \tau n !}{(r-1) !(n-r) !} \sum_{i=0}^{n-r}(-1)^{i}\left(\begin{array}{c}
n-r \\
i
\end{array}\right)\left[\Delta_{1}(p+\delta \tau(r+i), \tau(r+i))\right. \\
& \left.-\Delta_{1}(p+\delta \tau(r+i)+\delta, \tau(r+i))\right] .
\end{aligned}
$$

Remark 2. Putting $k=1$ in Eq. (7), we deduce the explicit expression for the single moment of lower record for GTL distribution in the form

$$
\alpha_{r}^{(p)}=\sum_{j=0}^{\infty} \frac{2^{-\frac{p}{\delta}-2 j}\left(\frac{p}{\delta}\right)}{j\left(\frac{p}{\delta}+j\right)} \operatorname{Bet}\left(\frac{p}{\delta}+j, j\right)\left(\frac{\tau \delta}{p+\tau \delta+\delta j}\right)^{r},
$$


as obtained by Zghoul (2011) for $\delta=1$.

Theorem 2 establishes a recurrence relation for single moments $\alpha_{r, n, m, k}^{(p)}$ from GTL distribution

Theorem 2. For $1 \leqslant r \leqslant n, k \geqslant 1$ and $m \geqslant-1$

$$
\alpha_{r-1, n, m, k}^{(p+\delta)}=\left(1+\frac{\delta}{p}\right) \alpha_{r-1, n, m, k}^{(p)}+\left(1+\frac{p+\delta}{2 \delta \tau \gamma_{r}}\right) \alpha_{r, n, m, k}^{(p+\delta)}-\left(\frac{p+\delta}{\tau \delta \gamma_{r}}+\frac{\delta}{p}+1\right) \alpha_{r, n, m, k}^{(p)} .
$$

Throughout, we follow the conventions that $\alpha_{0, n, m, k}^{(p)}=0$ for $n \geqslant 1$ and $\alpha_{r, n, m, k}^{(0)}=1$ for $1 \leqslant r \leqslant n$.

Proof From Eqs (3) and (4), we have

$$
2 \alpha_{r, n, m, k}^{(p)}-\alpha_{r, n, m, k}^{(p+\delta)}=\frac{2 \delta \tau C_{r-1}}{(r-1) !} \int_{0}^{1} y^{p-1}\left(1-y^{\delta}\right) F^{\gamma_{r}-1}(y) g_{m}^{r-1}(F(y)) f(y) \mathrm{d} y .
$$

Upon integrating by parts by treating $y^{p-1}\left(1-y^{\delta}\right)$ for integration and rest of the integrand for differentiation and simplification, we get the result given in Eq. (10).

Corollary 2. For the GTL distribution given in Eq. (1)

$$
\alpha_{1, n, m, k}^{(p)}=\left(1+\frac{p+\delta}{2 \delta \tau \gamma_{1}}\right) \alpha_{1, n, m, k}^{(p+\delta)}-\left(\frac{p+\delta}{\tau \delta \gamma_{1}}+\frac{\delta}{p}\right) \alpha_{1, n, m, k}^{(p)} .
$$

Remark 3. Setting $m=0, k=1$ in Eq. (10), the recurrence relation for the single moments of order statistics of the GTL distribution can be obtained as

$$
\begin{aligned}
\alpha_{n-r+2: n}^{(p+\delta)}= & \left(1+\frac{\delta}{p}\right) \alpha_{n-r+2: n}^{(p)}+\left(1+\frac{p+\delta}{2 \delta \tau(n-r+1)}\right) \alpha_{n-r+1: n}^{(p+\delta)} \\
& -\left(\frac{p+\delta}{\tau \delta(n-r+1)}+\frac{\delta}{p}+1\right) \alpha_{n-r+1: n}^{(p)} .
\end{aligned}
$$

Replacing $n-r+1$ by $r-1$, we get

$$
\alpha_{r: n}^{(p+\delta)}=\left(1+\frac{\delta}{p}\right) \alpha_{r: n}^{(p)}+\left(1+\frac{p+\delta}{2 \delta \tau(r-1)}\right) \alpha_{r-1: n}^{(p+\delta)}-\left(\frac{p+\delta}{\tau \delta(r-1)}+\frac{\delta}{p}+1\right) \alpha_{r-1: n}^{(p)},
$$

which verify the result of Zghoul (2010) for $r=r+1$ and $\delta=1$.

Remark 4. Setting $m=-1$ and $k \geqslant 1$ in Eq. (10), the relation for single moment of lower $k$ record values is deduced in the form

$$
\alpha_{r}^{(p+\delta)}=\left(\frac{2 \delta \tau k}{2 \delta \tau k+p+\delta}\right) \alpha_{r-1}^{(p+\delta)}+\left(\frac{2(p+\delta)\left(p+k \delta^{2} \tau\right)}{p(2 \delta \tau k+p+\delta)}\right) \alpha_{r}^{(p)}-\left(\frac{2 \delta \tau k(p+\delta)}{p(2 \tau \delta k+p+\delta)}\right) \alpha_{r-1}^{(p)} .
$$

and hence for lower records $(k=1)$

$$
\alpha_{r}^{(p+\delta)}=\left(\frac{2 \delta \tau}{2 \delta \tau+p+\delta}\right) \alpha_{r-1}^{(p+\delta)}+\left(\frac{2(p+\delta)\left(p+\delta^{2} \tau\right)}{p(2 \delta \tau+p+\delta)}\right) \alpha_{r}^{(p)}-\left(\frac{2 \delta \tau(p+\delta)}{p(2 \tau \delta+p+\delta)}\right) \alpha_{r-1}^{(p)},
$$

as obtained by Zghoul (2011) for $\delta=1$.

\subsection{Relations for product moments of dgos}

Here, first we present the explicit expressions and recurrence relations for rth and sth $\operatorname{dgos}, Y(r, n, m, k)$, $Y(s, n, m, k)$ of GTL distribution

Theorem 3. For $1 \leqslant r<s \leqslant n, n \geqslant 1, k=1,2, \ldots$ and $p, q=1,2, \ldots$, then the explicit expressions for product moments of pth and qth $\operatorname{dgos}, E\left[Y(r, s, n, m, k)^{(p, q)}\right]=\alpha_{r, s, n, m, k}^{(p, q)}$ is given by

$$
\alpha_{r, s, n, m, k}^{(p, q)}=\frac{(2 \delta \tau)^{2} C_{s-1}}{(r-1) !(s-r-1) !(m+1)^{s-2}} \sum_{i=1}^{r-1} \sum_{j=1}^{s-r-1}\left(-1^{i+j}\right)\left(\begin{array}{c}
r-1 \\
i
\end{array}\right)\left(\begin{array}{c}
s-r-1 \\
j
\end{array}\right)
$$




$$
\begin{aligned}
& \times\left[\Delta_{2}\left\{p+\tau \delta\left(\gamma_{r-i}-\gamma_{s-j}\right)-1, \tau\left(\gamma_{r-i}-\gamma_{s-j}\right)-1 ; q+\tau \delta\left(\gamma_{s-j}\right)-1, \tau \gamma_{s-j}-1\right\}\right. \\
& -\Delta_{2}\left\{p+\tau \delta\left(\gamma_{r-i}-\gamma_{s-j}\right)+\delta-1, \tau\left(\gamma_{r-i}-\gamma_{s-j}\right)-1 ; q+\tau \delta\left(\gamma_{s-j}\right)-1, \tau \gamma_{s-j}-1\right\} \\
& -\Delta_{2}\left\{p+\tau \delta\left(\gamma_{r-i}-\gamma_{s-j}\right)-1, \tau\left(\gamma_{r-i}-\gamma_{s-j}\right)-1 ; q+\tau \delta\left(\gamma_{s-j}\right)+\delta-1, \tau \gamma_{s-j}-1\right\} \\
& +\Delta_{2}\left\{p+\tau \delta\left(\gamma_{r-i}-\gamma_{s-j}\right)+\delta-1, \tau\left(\gamma_{r-i}-\gamma_{s-j}\right)-1 ;\right. \\
& \left.\left.q+\tau \delta\left(\gamma_{s-j}\right)+\delta-1, \tau \gamma_{s-j}-1\right\}\right]
\end{aligned}
$$

where $\Delta_{2}\left(p_{1}, p_{2}, q_{1}, q_{2}\right)$ is defined in Eq. (26).

Proof From Eq. (5), we have

$$
\begin{aligned}
\alpha_{r, s, n, m, k}^{(p, q)}= & \frac{C_{s-1}}{(r-1) !(s-r-1) !} \int_{0}^{1} \int_{0}^{y} y^{p} z^{q} F^{m}(y) F^{\gamma_{s}-1}(z) g_{m}^{r-1}(F(y)) \\
& \times\left[g_{m}(F(z))-g_{m}(F(y))\right]^{s-r-1} f(y) f(z) \mathrm{d} y \mathrm{~d} z \\
= & \frac{C_{s-1}}{(r-1) !(s-r-1) !(m+1)^{s-2}} \sum_{i=0}^{r-1} \sum_{j=0}^{s-r-1}(-1)^{i+j}\left(\begin{array}{c}
r-1 \\
i
\end{array}\right)\left(\begin{array}{c}
s-r-1 \\
j
\end{array}\right) \\
& \times \int_{0}^{1} \int_{0}^{y} y^{p} z^{q}[F(y)]^{\gamma_{r-i}-\gamma_{s-j}-1}[F(z)]^{\gamma_{s-j}-1} f(y) f(z) \mathrm{d} z \mathrm{~d} y \\
= & \frac{(2 \delta \tau)^{2} C_{s-1}}{(r-1) !(s-r-1) !(m+1)^{s-2}} \sum_{i=0}^{r-1} \sum_{j=0}^{s-r-1}(-1)^{i+j}\left(\begin{array}{c}
r-1 \\
i
\end{array}\right)\left(\begin{array}{c}
s-r-1 \\
j
\end{array}\right) \\
& \times \int_{0}^{1} \int_{0}^{y} y^{p+\tau \delta\left(\gamma_{r-i}-\gamma_{s-j}\right)-1}\left(2-y^{\delta}\right)^{\tau\left(\gamma_{r-i}-\gamma_{s-j}\right)-1} z^{q+\tau \delta \gamma_{s-j}-1} \\
& \times\left(2-z^{\delta}\right)^{\tau \gamma_{s-j}-1}\left(1-y^{\delta}-z^{\delta}-y^{\delta} z^{\delta}\right) \mathrm{d} z \mathrm{~d} y .
\end{aligned}
$$

The result follows by using Lemma 2 . The proof is complete.

Corollary 3. For $m=-1$ in Eq. (11), the explicit expression for the product moments of lower $k$ record values for the GTL distribution can be obtained by using L'Hospital's Rule as

$$
\alpha_{r, s, n,-1, k}^{(p, q)}=\sum_{i=0}^{\infty} \sum_{i=0}^{\infty} \frac{2^{-\frac{(p+q)}{\delta}-2(i+j)}\left(\frac{q}{\delta}\right)\left(\frac{p}{\delta}\right)}{i j\left(\frac{p}{\delta}+j\right)\left(\frac{q}{\delta}+j\right)}\left(\frac{k \delta \tau}{q+k \delta \tau+i \delta}\right)^{s-r}\left(\frac{k \delta \tau}{p+q+k \delta \tau+\delta(i+j)}\right)^{r} .
$$

Remark 5. If $m=0, k=1$ in Eq. (11), the we get exact explicit expression for the product moments of order statistics for GTL distribution

$$
\begin{aligned}
\alpha_{r, s: n}^{(p, q)}= & \frac{(2 \delta \tau)^{2} n !}{(r-1) !(s-r-1) !(n-s) !} \sum_{i=1}^{r-1} \sum_{j=1}^{s-r-1}\left(-1^{i+j}\right)\left(\begin{array}{c}
r-1 \\
i
\end{array}\right)\left(\begin{array}{c}
s-r-1 \\
j
\end{array}\right) \\
& \times\left[\Delta_{2}\{p+\tau \delta(s-r+i-j)-1, \tau(s-r+i-j)-1 ; q+\tau \delta(n-s+j+1)-1,\right. \\
& \tau(n-s+j+1)-1\}-\Delta_{2}\{p+\tau \delta(s-r+i-j)+\delta-1, \tau(s-r+i-j)-1 ; \\
& q+\tau \delta(n-s+j+1)-1, \tau(n-s+j+1)-1\}-\Delta_{2}\{p+\tau \delta(s-r+i-j)-1, \\
& \tau(s-r+i-j)-1 ; q+\tau \delta(n-s+j+1)+\delta-1, \tau(n-s+j+1)-1\} \\
& +\Delta_{2}\{p+\tau \delta(s-r+i-j)+\delta-1, \tau(s-r+i-j)-1 ; q+\tau \delta(n-s+j+1)+\delta-1, \\
& \tau(n-s+j+1)-1\}] .
\end{aligned}
$$

Remark 6. If $k=1$ in Eq. (12), then we get the lower record values of GTL distribution as

$$
\alpha_{r, s}^{(p, q)}=\sum_{i=0}^{\infty} \sum_{i=0}^{\infty} \frac{2^{-\frac{(p+q)}{\delta}-2(i+j)}\left(\frac{q}{\delta}\right)\left(\frac{p}{\delta}\right)}{i j\left(\frac{p}{\delta}+j\right)\left(\frac{q}{\delta}+j\right)}\left(\frac{\delta \tau}{q+\delta \tau+i \delta}\right)^{s-r}\left(\frac{\delta \tau}{p+q+\delta \tau+\delta(i+j)}\right)^{r} .
$$


Theorem 4. For $1 \leqslant r<s \leqslant n, n \geqslant 1, k=1,2, \ldots$ and $p, q=1,2, \ldots$

$$
\left(1+\frac{q+\delta}{2 \tau \delta \gamma_{s}}\right) \alpha_{r, s, n, m, k}^{(p, q+\delta)}=\alpha_{r, s-1, n, m, k}^{(p, q+\delta)}+(q+\delta)\left(\frac{1}{\delta \tau \gamma_{s}}+\frac{\delta}{q}\right) \alpha_{r, s, n, m, k}^{(p, q)}-\left(1+\frac{\delta}{q}\right) \alpha_{r, s-1, n, m, k}^{(p, q+\delta)} .
$$

Proof From Eqs (3) and (5), we have

$$
\begin{aligned}
2 \alpha_{r, s, n, m, k}^{(p, q)}-\alpha_{r, s, n, m, k}^{(p, q+\delta)}= & \frac{C_{s-1}}{(r-1) !(s-r-1) !} \int_{0}^{1} \int_{0}^{y} y^{p} z^{q-1}[F(y)]^{m} f(y) g_{m}^{r-1}(F(y)) \\
& \times\left(1-z^{\delta}\right)\left[h_{m}(F(z))-h_{m}(F(y))\right]^{s-r-1}[F(z)]^{\gamma_{s}} \mathrm{~d} y \mathrm{~d} z
\end{aligned}
$$

Integrating by parts and treating $z^{q-1}\left(1-z^{\delta}\right)$ for integration and rest of the integrand for differentiation and after simplification, we get the result given in Theorem 4 .

Remark 7. Setting $m=0, k=1$ in Eq. (13), we get moments of order statistics

$$
\alpha_{r, s: n}^{(p, q+\delta)}=\left(1+\frac{q+\delta}{2 \tau \delta(r-1)}\right) \alpha_{r-1, s: n}^{(p, q+\delta)}-(q+\delta)\left(\frac{1}{\delta \tau(r-1)}+\frac{\delta}{q}\right) \alpha_{r-1, s: n}^{(p, q)}+\left(1+\frac{\delta}{q}\right) \alpha_{r, s: n}^{(p, q+\delta)} .
$$

Which verifies the result of Kumar (2012) for $\delta=1$.

Remark 8. For $m=-1$ in Eq. (13), we obtain relation for lower $k$ record values

$$
\alpha_{r, s-1}^{(p, q+\delta)}=\left(1+\frac{q+\delta}{2 \tau \delta k}\right) \alpha_{r, s}^{(p, q+\delta)}+(q+\delta)\left(\frac{1}{\delta \tau k}-\frac{\delta}{q}\right) \alpha_{r, s: n}^{(p, q)}+\left(1+\frac{\delta}{q}\right) \alpha_{r, s-1}^{(p, q+\delta)} .
$$

and hence for lower records $k=1$.

$$
\alpha_{r, s-1}^{(p, q+\delta)}=\left(1+\frac{q+\delta}{2 \tau \delta}\right) \alpha_{r, s}^{(p, q+\delta)}+(q+\delta)\left(\frac{1}{\delta \tau}-\frac{\delta}{q}\right) \alpha_{r, s: n}^{(p, q)}+\left(1+\frac{\delta}{q}\right) \alpha_{r, s-1}^{(p, q+\delta)} .
$$

\section{Estimation based on dgos}

\subsection{Maximum likelihood estimation}

Let $Y_{1}, Y_{2}, \ldots, Y_{n}$ be the random variables of size $n$ and $Y(1, n, m, k), Y(2, n, m, k), \ldots, Y(n, m, m, k)$ be the corresponding dgos drawn from the GTL distribution with pdf and cdf given by Eqs (1) and (2), respectively. By using Eqs (1) and (2), the likelihood function can be written as follows

$$
L(\tau, \delta \mid \mathbf{y})=(2 \delta \tau)^{n} k \prod_{j=1}^{n-1} \gamma_{j} \prod_{i=1}^{n-1} y_{i}^{\tau \delta\left(m_{i}+1\right)-1}\left(2-y_{i}^{\delta}\right)^{\tau\left(m_{i}+1\right)-1} y_{n}^{\tau \delta k-1}\left(2-y_{n}^{\delta}\right)^{\tau k-1} \prod_{i=1}^{n}\left(1-y_{i}^{\delta}\right) .
$$

The natural logarithm of the likelihood function $\ell(\tau, \delta)=\log L(\tau, \delta \mid \mathbf{y})$ without constant term is given by

$$
\begin{aligned}
\ell(\tau, \delta)= & n \log \tau+n \log \delta+\sum_{i=1}^{n} \log \left(1-y_{i}^{\delta}\right)+\sum_{i=1}^{n-1}\left(\tau\left(m_{i}+1\right)-1\right) \log \left(2-y_{i}^{\delta}\right) \\
& +\sum_{i=1}^{n-1}\left(\tau \delta\left(m_{i}+1\right)-1\right) \log y_{i}+(\tau k-1) \log \left(2-y_{n}^{\delta}\right)+(\tau \delta k-1) \log y_{n} .
\end{aligned}
$$

By differentiating Eq. (15) partially with respect $\tau$ and $\delta$ and equate the results to zero, we have

$$
\frac{\partial \ell(\tau, \delta)}{\partial \tau}=\frac{n}{\tau}+\sum_{i=1}^{n-1}\left(m_{i}+1\right) \log \left[y_{i}^{\delta}\left(2-y_{i}^{\delta}\right)\right]+k \log \left[y_{n}^{\delta}\left(2-y_{n}^{\delta}\right)\right]=0
$$

and

$$
\frac{\partial \ell(\tau, \delta)}{\partial \delta}=\frac{n}{\delta}-\sum_{i=1}^{n} \frac{y_{i}^{\delta} \log y_{i}}{\left(1-y_{i}^{\delta}\right)}-\sum_{i=1}^{n-1} \frac{\left(\tau\left(m_{i}+1\right)-1\right) y_{i}^{\delta} \log y_{i}}{\left(2-y_{i}^{\delta}\right)}+\sum_{i=1}^{n-1} \tau\left(m_{i}+1\right) \log y_{i}
$$




$$
-\frac{(\tau k-1) y_{n}^{\delta} \log y_{n}}{\left(2-y_{n}^{\delta}\right)}+\tau k \log y_{n}=0
$$

The maximum likelihood estimator (MLE) of $\tau$ as a function of $\delta$, can be obtained as

$$
\hat{\tau}(\delta)=-\frac{n}{\psi(y ; \delta)},
$$

where

$$
\psi(y ; \delta)=\sum_{i=1}^{n-1}\left(m_{i}+1\right) \log \left[y_{i}^{\delta}\left(2-y_{i}^{\delta}\right)\right]+k \log \left[y_{n}^{\delta}\left(2-y_{n}^{\delta}\right)\right] .
$$

The MLE of $\delta$ is the solution of

$$
\begin{aligned}
\frac{n}{\delta}- & \sum_{i=1}^{n} \frac{z_{i}}{1-y_{i}^{\delta}}-\sum_{i=1}^{n-1} \frac{\left(\hat{\tau}(\delta)\left(m_{i}+1\right)-1\right) z_{i}}{2-y_{i}^{\delta}}+\sum_{i=1}^{n-1} \hat{\tau}(\delta)\left(m_{i}+1\right) \log y_{i} \\
& -\frac{(\hat{\tau}(\delta) k-1) z_{n}}{2-y_{n}^{\delta}}+\tau \log y_{n}=0
\end{aligned}
$$

where $z_{i}=y_{i}^{\delta} \log y_{i}, i=1, \ldots, n$. It is noted that the MLE of the parameter $\delta$ cannot be obtained in closed form, therefore, a numerical techniques can be used to solve Eq. (15) to obtain the MLE of $\delta$. Once the MLE of $\delta$ is obtained, the MLE of $\tau$ can be obtained directly from Eq. (14).

To construct the 100(1- $\xi) \%$ two-sided ACIs for the unknown parameters $\tau$ and $\delta$, the Fisher's information matrix must be obtained. Asymptotic variance-covariance (V-C) matrix of the MLEs $\hat{\Theta}=(\hat{\tau}, \hat{\delta})^{\mathrm{T}}$ can be obtained by inverting Fisher information matrix, $\mathbf{I}(\Theta)$ in the form

$$
\mathbf{I}_{i j}(\Theta)=E\left[-\left(\partial^{2} \ell(\Theta \mid \mathbf{x})\right) / \partial \Theta^{2}\right], i, j=1,2 .
$$

Practically, by dropping the expectation operator $E$ and replacing $\Theta$ by their MLE $\hat{\Theta}$, we get the approximate asymptotic V-C matrix for the MLEs, see Cohen (1965), as

$$
\mathbf{I}^{-1}(\hat{\tau}, \hat{\delta}) \cong\left[\begin{array}{ll}
-\mathcal{L}_{\tau \tau}-\mathcal{L}_{\tau \delta} \\
-\mathcal{L}_{\delta \tau}-\mathcal{L}_{\delta \delta}
\end{array}\right]_{(\tau=\hat{\tau}, \delta=\hat{\delta})}^{-1}=\left[\begin{array}{ll}
\hat{\mathbf{V}}_{\hat{\hat{\tau}} \hat{\tau}} & \hat{\mathbf{V}}_{\hat{\hat{\delta}} \hat{\delta}} \\
\hat{\mathbf{V}}_{\hat{\delta} \hat{\tau}} & \hat{\mathbf{V}}_{\hat{\delta} \hat{\delta}}
\end{array}\right] .
$$

From Eq. (18), the Fisher's elements will be

$$
\begin{aligned}
& \frac{\partial^{2} \ell(\tau, \delta)}{\partial \tau^{2}}=-\frac{n}{\tau^{2}}, \\
& \frac{\partial^{2} \ell(\tau, \delta)}{\partial \delta^{2}}=-\frac{n}{\delta^{2}}-\sum_{i=1}^{n} \frac{y_{i}^{\delta}\left(\log y_{i}\right)^{2}}{\left(1-y_{i}^{\delta}\right)^{2}}-\sum_{i=1}^{n-1} \frac{2\left(\tau\left(m_{i}+1\right)-1\right) y_{i}^{\delta}\left(\log y_{i}\right)^{2}}{\left(2-y_{i}^{\delta}\right)^{2}}-\frac{2(\tau k-1) y_{n}^{\delta}\left(\log y_{n}\right)^{2}}{\left(2-y_{n}^{\delta}\right)^{2}},
\end{aligned}
$$

and

$$
\frac{\partial^{2} \ell(\tau, \delta)}{\partial \tau \partial \delta}=\sum_{i=1}^{n-1} \frac{2\left(m_{i}+1\right)\left(1-y_{i}^{\delta}\right) \log y_{i}}{\left(2-y_{i}^{\delta}\right)}+\frac{2 k\left(1-y_{n}^{\delta}\right) \log y_{n}}{\left(2-y_{n}^{\delta}\right)}
$$

Under some regularity conditions, the asymptotic normality of MLEs $\hat{\Theta}=(\hat{\tau}, \hat{\delta})^{\mathrm{T}}$ is approximately bivariate normal as $\hat{\Theta} \sim N\left(\Theta, \mathbf{I}^{-1}(\hat{\Theta})\right)$. Hence, using the large sample theory, the $100(1-\xi) \%$ two-sided ACIs for $\tau$ and $\delta$ can be obtained, respectively, by

$$
\hat{\tau} \mp z_{\xi / 2} \sqrt{\hat{\mathbf{V}}_{\hat{\tau} \hat{\tau}}} \text { and } \hat{\delta} \mp z_{\xi / 2} \sqrt{\hat{\mathbf{V}}_{\hat{\delta} \hat{\delta}}} \text {, }
$$

where $\hat{\mathbf{V}}_{\hat{\tau} \hat{\tau}}$ and $\hat{\mathbf{V}}_{\hat{\delta} \hat{\delta}}$ are the main diagonal elements of Eq. (18), respectively, and $z_{\xi / 2}$ is the percentile of the standard normal distribution with upper probability $(\xi / 2)$ th. 


\subsection{Bayesian estimation}

In this subsection, we focus on obtaining the Bayes estimates of the unknown model parameters $\tau$ and $\delta$ under dgos with respect to SEL function. Prior distributions play an essential role for derivation of the Bayes estimators. There is no clear method on choosing priors for a particular problem. We refer to Arnold and Press (1983) for more details on this. In the premise of above arguments, we consider the Bayesian estimation under the assumption that the random variables $\tau$ and $\delta$ have independent gamma priors. Let $\tau \sim \operatorname{Gamma}\left(a_{1}, b_{1}\right)$ and $\delta \sim \operatorname{Gamma}\left(a_{2}, b_{2}\right)$. The Gamma distribution is flexible in nature, it can provide for different shapes based on parameter values and thus it can be considered as suitable priors of model parameters. See for more details Kundu and Pradhan (2009), Dey et al. (2015, 2016a, 2016b). Thus the joint prior density can be written as

$$
g(\tau, \delta) \propto \tau^{a_{1}-1} \delta^{a_{2}-1} e^{-\left(b_{1} \tau+b_{2} \delta\right)}, a_{1}, a_{2}, b_{1}, b_{2}>0,
$$

Now, we can write the joint posterior distribution of $\tau$ and $\delta$ based on Eqs (12) and (16) as follows

$$
\begin{aligned}
g(\tau, \delta \mid \mathbf{y})= & C^{-1} \delta^{n+a_{2}-1} \tau^{n+a_{1}-1} \prod_{i=1}^{n-1} y_{i}^{\tau \delta\left(m_{i}+1\right)-1}\left(2-y_{i}^{\delta}\right)^{\tau\left(m_{i}+1\right)-1} y_{n}^{\tau \delta k-1}\left(2-y_{n}^{\delta}\right)^{\tau k-1} \\
& \times e^{-\left(b_{1} \tau+b_{2} \delta\right)} \prod_{i=1}^{n}\left(1-y_{i}^{\delta}\right)
\end{aligned}
$$

where $C$ is the normalized constant and given by $C=\int_{0}^{\infty} \int_{0}^{\infty} g(\tau, \delta \mid \mathbf{y}) \mathrm{d} \tau \mathrm{d} \delta$. The Bayes estimator based on any loss function of $\tau$ and $\delta$, say $\varphi(\tau, \delta)$, can be obtained as follows

$$
\tilde{\varphi}(\tau, \delta)=\int_{0}^{\infty} \int_{0}^{\infty} \varphi(\tau, \delta) g(\tau, \delta \mid \mathbf{y}) \mathrm{d} \tau \mathrm{d} \delta .
$$

From Eq. (18) we can observe that it is not possible to obtain the Bayes estimators of $\tau$ and $\delta$ analytically. Therefore, we suggest to use the MCMC procedure to generate samples from Eq. (17) and use the generated samples to obtain the Bayes estimates of $\tau$ and $\delta$ using SEL function. To use the MCMC procedure, we first need to obtain the full conditional distributions of $\tau$ and $\delta$ as follows

$$
g_{\tau}(\tau \mid \delta, \mathbf{y}) \propto \tau^{n+a_{1}-1} e^{-b_{1} \tau} \prod_{i=1}^{n-1} y_{i}^{\tau \delta\left(m_{i}+1\right)}\left(2-y_{i}^{\delta}\right)^{\tau\left(m_{i}+1\right)-1} y_{n}^{\tau \delta k}\left(2-y_{n}^{\delta}\right)^{\tau k-1}
$$

and

$$
g_{\delta}(\delta \mid \tau, \mathbf{y}) \propto \delta^{n+a_{2}-1} e^{-b_{2} \delta} \prod_{i=1}^{n-1} y_{i}^{\tau \delta\left(m_{i}+1\right)}\left(2-y_{i}^{\delta}\right)^{\tau\left(m_{i}+1\right)-1} y_{n}^{\tau \delta k}\left(2-y_{n}^{\delta}\right)^{\tau k-1} \prod_{i=1}^{n}\left(1-y_{i}^{\delta}\right) .
$$

It is observed that the conditional posterior distributions of the unknown parameters $\tau$ and $\delta$ as in Eqs (22) and (23), respectively, cannot be reduced analytically to well-known distributions. Therefore, the Metropolis-Hastings (M-H) algorithm using normal distribution as a proposal distribution is used to generate random samples from Eqs (23) and (23) and in turn to obtain the Bayes estimates (BEs) and corresponding HPD credible intervals. To carried out the M-H algorithm procedure, do following steps for sample generation process:

Step 1: Set an initial values $\tau^{(0)}$ and $\delta^{(0)}$.

Step 2: Set $j=1$.

Step 3: Generate $\tau^{(j)}$ and $\delta^{(j)}$ from Eqs (22) and (23) with normal proposal distributions $N\left(\tau^{(j-1)}, \mathbf{V}_{\tau \tau}\right)$ and $N\left(\delta^{(j-1)}, \mathbf{V}_{\delta \delta}\right)$, respectively, as

(a) Generate $\tau^{*}$ from $N\left(\tau^{(j-1)}, \mathbf{V}_{\tau \tau}\right)$ and $\delta^{*}$ from $N\left(\delta^{(j-1)}, \mathbf{V}_{\delta \delta}\right)$.

(b) Obtain

$$
\phi_{\tau}=\left\{1, \frac{g_{\tau}\left(\tau^{*} \mid \delta^{(j-1)}, \mathbf{y}\right)}{g_{\tau}\left(\tau^{(j-1)} \mid \delta^{(j-1)}, \mathbf{y}\right)}\right\} \text { and } \phi_{\delta}=\left\{1, \frac{g_{\delta}\left(\delta^{*} \mid \tau^{(j)}, \mathbf{y}\right)}{g_{\delta}\left(\delta^{(j-1)} \mid \tau^{(j)}, \mathbf{y}\right)}\right\}
$$

(c) Generate $u_{1}$ and $u_{2}$ from $U(0,1)$.

(d) If $u_{1} \leqslant \phi_{\tau}$, set $\tau^{(j)}=\tau^{*}$, else set $\tau^{(j)}=\tau^{(j-1)}$.

(e) If $u_{2} \leqslant \phi_{\delta}$, set $\delta^{(j)}=\delta^{*}$, else set $\delta^{(j)}=\delta^{(j-1)}$. 
Step 4: Set $j=j+1$.

Step 5: Redo steps 3-4 for $N$ times to obtain $\left(\tau^{(j)}, \delta^{(j)}\right), j=1,2, \ldots, N$.

To construct the HPD credible intervals of $\tau$ and $\delta$, order the MCMC sample of $\tau^{(j)}$ and $\delta^{(j)}$ for $j=1,2, \ldots, N$ as $\tau_{(1)}, \tau_{(2)}, \ldots, \tau_{(N)}$ and $\delta_{(1)}, \delta_{(2)}, \ldots, \delta_{(N)}$. Using the method proposed by Chen and Shao (1999), the $100(1-\xi) \%$ two-sided HPD credible interval for any parametric function of $\tau$ and $\delta$, say $(\varphi(\tau, \delta))$, is given by

$$
\left[\varphi_{\left(j^{*}\right)}, \varphi\left(j^{*}+(1-\xi) N\right)\right]
$$

where $j^{*}$ is chosen such that

$$
\varphi_{\left(j^{*}+(1-\xi) N\right)}-\varphi_{\left(j^{*}\right)}=\min _{1 \leqslant j \leqslant \xi N}\left(\varphi_{(j+(1-\xi) N)}-\varphi_{(j)}\right), j^{*}=1,2, \ldots, N .
$$

Here $[x]$ denotes the largest integer less than or equal to $x$. Hence, the HPD credible interval is that interval which has the shortest length. The first simulated varieties, $M$, of the algorithm may be biased by the initial value, therefore, usually discarded at the beginning of implementation of analysis (burn-in period) to remove the effect of the initial selection. Thus, the BEs of $\varphi(\tau, \delta)$ based on SEL function is given by $\tilde{\varphi}(\tau, \delta)=\sum_{j=M+1}^{N} \varphi^{(j)} /(N-M)$.

\section{Monte carlo simulation}

In this section, a Monte Carlo simulation is conducted to examine and compare the performance of the proposed maximum likelihood and Bayes estimators of the unknown parameters $\tau$ and $\delta$ based on different sample sizes $n$. Comparison between different proposed estimators are made with respect to their RMSE and RAB as well as AL and $\mathrm{CP}$ values of point and interval estimators, respectively. For this purpose, we introduced the following algorithm:

Step 1: Set two arbitrarily true values of $\tau$ and $\delta$ such as $(\delta, \tau)=(0.1,0.5)$ and $(\delta, \tau)=(0.2,0.7)$.

Step 2: Set hyper-parameters values of $a_{i}, b_{i}, i=1,2$, such as $\left(a_{1}, a_{2}\right)=(1,1),\left(b_{1}, b_{2}\right)=(10,2)$ for $(\delta, \tau)=$ $(0.1,0.5)$ and $\left(a_{1}, a_{2}\right)=(1,7),\left(b_{1}, b_{2}\right)=(5,10)$ for $(\delta, \tau)=(0.2,0.7)$. Here, the values of hyperparameters are chosen to satisfy the prior mean become the expected value of the corresponding parameter.

Step 3: For $n=20,40,60,80$ and 100, the samples from GTL distribution can be generated from Eq. (2), $X_{i}=\left(1-\sqrt{1-u_{i}^{1 / \tau}}\right)^{1 / \delta}$, where $u_{i}$ is uniformly distributed random variate.

Step 4: Two special cases of $d g o s$ are considered, the first is the order statistics (OS) by taking $m_{i}=0$ and $k=1$, the second is the lower record (LR) by setting $m_{i}=-1$ and $k=1$. Therefore, OS and LR data are generated from GTL distribution.

Step 5: Using Newton-Raphson iterative method, the MLEs $\hat{\tau}$ and $\hat{\delta}$ of $\tau$ and $\delta$, respectively, which maximized Eq. (14), and corresponding ACIs are computed based on OS and LR values.

Step 6: Using Metropolis-Hastings within Gibbs algorithm described in Subsection 4.2, the MCMC Bayes estimates $\tilde{\tau}$ and $\tilde{\delta}$ of $\tau$ and $\delta$, respectively, with corresponding HPD credible intervals under the SEL function are computed by generating 12,000 MCMC samples and discard the first 2,000 iterations as burn-in.

Step 7: Repeat Steps 3-6 for 5,000 times and obtain the average estimates (AEs) for any parameteric function of $\tau$ and $\delta$, say $(\varphi(\tau, \delta))$ under SEL loss function with their RMSE and RAB values, respecively, as

$$
\overline{\hat{\varphi}}=\frac{1}{S} \sum_{i=1}^{S} \hat{\varphi}_{i}, \operatorname{RMSE}(\hat{\varphi})=\sqrt{\frac{1}{S} \sum_{i=1}^{S}\left(\hat{\varphi}_{i}-\varphi\right)^{2}}, \text { and } \operatorname{RAB}(\hat{\varphi})=\frac{1}{S} \sum_{i=1}^{S} \frac{\left|\hat{\varphi}_{i}-\varphi\right|}{\varphi},
$$

where $S$ is the number of generated sequence data and $\hat{\varphi}$ is the MLE or BE of the true value of the parametric function $\varphi=(\delta, \tau)$. The computational results are reported in Tables 1-3.

Extensive computations were performed using $\mathcal{R}$ statistical programming language software with mainly two useful statistical packages are 'CODA' package proposed by Plummer et al. (2006), and 'maxLik' package, which using Newton-Raphson method of maximization in the computations, proposed by Henningsen and Toomet (2011).

From Tables 1-3, we are able to make the following observations. The performances of the proposed estimates of $\tau$ and $\delta$ in terms of their RMSEs and RABs become better as $n$ increases, as expected. 
Table 1

The AEs of $\delta$ with their respective RMSEs and RABs in parenthesis

\begin{tabular}{|c|c|c|c|c|c|}
\hline \multirow[t]{2}{*}{$(\delta, \tau)$} & \multirow[t]{2}{*}{$n$} & \multicolumn{2}{|c|}{ MLE } & \multicolumn{2}{|c|}{$\mathrm{BE}$} \\
\hline & & OS & LR & OS & LR \\
\hline \multirow[t]{9}{*}{$(0.1,0.5)$} & 20 & $\begin{array}{c}0.3965 \\
(2.1899 .3 .6538)\end{array}$ & $\begin{array}{c}0.0946 \\
(0.02390 .0538)\end{array}$ & $\begin{array}{c}0.0801 \\
(0.0240,0.2050)\end{array}$ & $\begin{array}{c}0.4120 \\
(0.3296,3.1202)\end{array}$ \\
\hline & 40 & 0.2758 & 0.0972 & 0.0908 & 0.4856 \\
\hline & & $(1.8928,2.2698)$ & $(0.0172,0.0283)$ & $(0.0143,0.1181)$ & $(0.4037,3.8564)$ \\
\hline & 60 & 0.1747 & 0.0984 & 0.0896 & 0.5699 \\
\hline & & $(1.1252,1.1715)$ & $(0.0127,0.0156)$ & $(0.0142,0.1182)$ & $(0.4838,4.6988)$ \\
\hline & 80 & 0.1311 & 0.0987 & 0.1009 & 0.6847 \\
\hline & & $(0.4632,0.6936)$ & $(0.0115,0.0129)$ & $(0.0101,0.0808)$ & $(0.6045,5.8472)$ \\
\hline & 100 & 0.1171 & 0.0990 & 0.1047 & 0.7715 \\
\hline & & $(0.1680,0.5109)$ & $(0.0104,0.0105)$ & $(0.0099,0.0818)$ & $(0.7032,6.7148)$ \\
\hline \multirow[t]{10}{*}{$(0.2,0.7)$} & 20 & 0.8413 & 0.1884 & 0.1924 & 0.5264 \\
\hline & & $(7.9099,3.8938)$ & $(0.0511,0.0582)$ & $(0.0357,0.1524)$ & $(0.3347,1.6321)$ \\
\hline & 40 & 0.4543 & 0.1950 & 0.1962 & 0.5160 \\
\hline & & $(3.5710,1.8081)$ & $(0.0333,0.0252)$ & $(0.0213,0.0863)$ & $(0.3332,1.5801)$ \\
\hline & 60 & 0.3219 & 0.1970 & 0.2020 & 0.6019 \\
\hline & & $(1.8096,1.0412)$ & $(0.0256,0.0151)$ & $(0.0224,0.0847)$ & $(0.4384,2.0093)$ \\
\hline & 80 & 0.2344 & 0.1973 & 0.1950 & 0.6485 \\
\hline & & $(0.6286,0.5575)$ & $(0.0243,0.0136)$ & $(0.0157,0.0645)$ & $(0.4655,2.2424)$ \\
\hline & 100 & 0.2243 & 0.1981 & 0.1930 & 0.7917 \\
\hline & & $(0.2461,0.4669)$ & $(0.0200,0.0093)$ & $(0.0141,0.0565)$ & $(0.6047,2.9584)$ \\
\hline
\end{tabular}

Table 2

The AEs of $\tau$ with their respective RMSEs and RABs in parenthesis

\begin{tabular}{|c|c|c|c|c|c|}
\hline \multirow[t]{2}{*}{$(\delta, \tau)$} & \multirow[t]{2}{*}{$n$} & \multicolumn{2}{|c|}{ MLE } & \multicolumn{2}{|c|}{$\mathrm{BE}$} \\
\hline & & OS & LR & OS & LR \\
\hline \multirow[t]{10}{*}{$(0.1,0.5)$} & 20 & 17.438 & 0.5045 & 0.5188 & 0.6678 \\
\hline & & $(87.216,34.331)$ & $(0.0201,0.0090)$ & $(0.0355,0.0593)$ & $(0.1769,0.3357)$ \\
\hline & 40 & 3.1199 & 0.5024 & 0.5112 & 0.6333 \\
\hline & & $(32.639,5.6498)$ & $(0.0148,0.0049)$ & $(0.0209,0.0327)$ & $(0.1413,0.2665)$ \\
\hline & 60 & 1.0034 & 0.5014 & 0.4883 & 0.5382 \\
\hline & & $(3.6558,1.3772)$ & $(0.0112,0.0028)$ & $(0.0190,0.0314)$ & $(0.0481,0.0771)$ \\
\hline & 80 & 0.8228 & 0.5011 & 0.4887 & 0.5729 \\
\hline & & $(1.8427,0.9821)$ & $(0.0102,0.0023)$ & $(0.0176,0.0282)$ & $(0.0846,0.1458)$ \\
\hline & 100 & 0.7161 & 0.5009 & 0.5070 & 0.5852 \\
\hline & & $(1.1628,0.7523)$ & $(0.0089,0.0018)$ & $(0.0156,0.0249)$ & $(0.0998,0.1713)$ \\
\hline \multirow[t]{10}{*}{$(0.2,0.7)$} & 20 & 32.329 & 0.7100 & 0.5882 & 0.8123 \\
\hline & & $(128.72,45.638)$ & $(0.0438,0.0142)$ & $(0.1160,0.1597)$ & $(0.1300,0.1633)$ \\
\hline & 40 & 5.5303 & 0.7045 & 0.7056 & 0.8130 \\
\hline & & $(36.371,7.2950)$ & $(0.0295,0.0064)$ & $(0.0185,0.0213)$ & $(0.1307,0.1614)$ \\
\hline & 60 & 2.0287 & 0.7027 & 0.7074 & 0.6988 \\
\hline & & $(14.341,2.2711)$ & $(0.0228,0.0038)$ & $(0.0178,0.0205)$ & $(0.0421,0.0559)$ \\
\hline & 80 & 1.2408 & 0.7024 & 0.7008 & 0.7680 \\
\hline & & $(2.6203,1.1009)$ & $(0.0216,0.0035)$ & $(0.0103,0.0124)$ & $(0.0731,0.0972)$ \\
\hline & 100 & 1.0462 & 0.7016 & 0.7080 & 0.7540 \\
\hline & & $(1.9061,0.8135)$ & $(0.0177,0.0023)$ & $(0.0160,0.0175)$ & $(0.0593,0.0772)$ \\
\hline
\end{tabular}

Also, the ALs of ACI/HPD credible intervals narrow down as $n$ increases, as expected. It can be seen that, the BEs using gamma informative prior are better as they include prior information than MLEs in respect of their RMSEs and RABs. It is also observed that the RMSEs and RABs associated with the MLEs of the model parameters $\tau$ and $\delta$ increase based on OS while that decrease based on LR.

Further, the RMSEs and RABs associated with the BEs of $\delta$ increase based on LR while that decrease based on OS. Also, the RMSEs and RABs associated with proposed estimators increases, when the parameter value of $\tau$ and $\delta$ increases.

For interval estimates, the 95\% HPD credible intervals are better than ACIs in respect of their ALs and CPs. Moreover, the ACIs of $\tau$ and $\delta$ become even better besed on LR than OS in respect of their CPs. Furthermore, the 
Table 3

The ALs of $\tau$ and $\delta$ with their CPs in parentheses

\begin{tabular}{|c|c|c|c|c|c|c|c|c|c|}
\hline \multirow[t]{3}{*}{$(\delta, \tau)$} & \multirow[t]{3}{*}{$n$} & \multicolumn{4}{|c|}{$\tau$} & \multicolumn{4}{|c|}{$\delta$} \\
\hline & & \multicolumn{2}{|c|}{ ACI } & \multicolumn{2}{|c|}{ HPD } & \multicolumn{2}{|c|}{ ACI } & \multicolumn{2}{|c|}{ HPD } \\
\hline & & OS & LR & OS & LR & OS & LR & OS & LR \\
\hline \multirow[t]{9}{*}{$(0.1,0.5)$} & 20 & $\begin{array}{c}283.90 \\
(0.8678)\end{array}$ & $\begin{array}{c}0.4422 \\
(0.9995)\end{array}$ & $\begin{array}{c}0.1054 \\
(0.9900)\end{array}$ & $\begin{array}{c}0.1875 \\
(0.9980)\end{array}$ & $\begin{array}{c}3.9013 \\
(0.7652)\end{array}$ & $\begin{array}{c}0.0819 \\
(0.9490)\end{array}$ & $\begin{array}{c}0.0466 \\
(0.9766)\end{array}$ & $\begin{array}{c}0.3047 \\
(0.9925)\end{array}$ \\
\hline & 40 & 31.758 & 0.3114 & 0.0636 & 0.1384 & 1.9543 & 0.0598 & 0.0424 & 0.3484 \\
\hline & & $(0.8930)$ & $(0.9995)$ & $(0.9720)$ & $(0.9731)$ & $(0.8334)$ & $(0.9728)$ & $(0.9660)$ & $(0.9525)$ \\
\hline & 60 & 5.1739 & 0.2537 & 0.0517 & 0.0887 & 0.8119 & 0.0495 & 0.0370 & 0.4053 \\
\hline & & $(0.9080)$ & $(0.9997)$ & $(0.9762)$ & (0.9799) & $(0.8658)$ & $(0.9848)$ & $(0.9754)$ & (0.9997) \\
\hline & 80 & 2.8548 & 0.2196 & 0.0576 & 0.1306 & 0.4560 & 0.0431 & 0.0388 & 0.4801 \\
\hline & & $(0.9178)$ & (0.9998) & $(0.9880)$ & $(0.9535)$ & $(0.8778)$ & $(0.9874)$ & $(0.9849)$ & $(0.9877)$ \\
\hline & 100 & 2.0738 & 0.1963 & 0.0492 & 0.1464 & 0.2787 & 0.0387 & 0.0334 & 0.6752 \\
\hline & & $(0.9236)$ & (0.9999) & $(0.9555)$ & $(0.9830)$ & $(0.8914)$ & $(0.9898)$ & $(0.9798)$ & $(0.9967)$ \\
\hline \multirow[t]{10}{*}{$(0.2,0.7)$} & 20 & 581.67 & 0.6201 & 0.1204 & 0.2123 & 9.0819 & 0.1630 & 0.1081 & 0.2381 \\
\hline & & $(0.8724)$ & $(0.9990)$ & $(0.9696)$ & $(0.9760)$ & $(0.7784)$ & $(0.9480)$ & $(0.9633)$ & $(0.9958)$ \\
\hline & 40 & 66.834 & 0.4397 & 0.0714 & 0.1812 & 3.1801 & 0.1200 & 0.0754 & 0.3243 \\
\hline & & $(0.8978)$ & (0.9998) & $(0.9692)$ & $(0.9660)$ & $(0.8328)$ & $(0.9772)$ & $(0.9923)$ & $(0.9887)$ \\
\hline & 60 & 16.072 & 0.3557 & 0.0634 & 0.1192 & 1.5496 & 0.0990 & 0.0896 & 0.5180 \\
\hline & & $(0.9018)$ & (0.9998) & $(0.9690)$ & $(0.9652)$ & $(0.8660)$ & $(0.9860)$ & $(0.9858)$ & $(0.9922)$ \\
\hline & 80 & 4.8497 & 0.3078 & 0.0355 & 0.0903 & 0.5831 & 0.0861 & 0.0545 & 0.4193 \\
\hline & & $(0.9172)$ & $(0.9946)$ & $(0.9595)$ & $(0.9605)$ & $(0.8822)$ & $(0.9874)$ & $(0.9823)$ & $(0.9932)$ \\
\hline & 100 & 3.2833 & 0.2750 & 0.0496 & 0.0848 & 0.5018 & 0.0774 & 0.0469 & 0.4310 \\
\hline & & $(0.9192)$ & $(0.9914)$ & $(0.9727)$ & $(0.9649)$ & $(0.8932)$ & $(0.9914)$ & $(0.9700)$ & $(0.9958)$ \\
\hline
\end{tabular}

Table 4

Lower record data from data 1

\begin{tabular}{lllllllll}
\hline 0.083 & 0.025 & 0.023 & 0.017 & 0.015 & 0.008 & 0.007 & 0.005 & 0.001
\end{tabular}

CPs of the HPD credible intervals of $\tau$ and $\delta$ based on OS and LR, are very close to each other, as shown in Table 3 . Therefore, we recommend the Bayesian point and interval estimation of the unknown model parameters of GTL distribution using $\mathrm{M}-\mathrm{H}$ algorithm.

\section{Real-life data analysis}

In this section, we analyze two real data sets to illustrate our established results. The first one based on lower record (LR) and the second one based on order statistics (OS). It is known that OR and LR can be obtained from the dgos as a special case, therefore, the estimators and confidence intervals of the GTL distribution based on LR and OS can be obtained directly from Section 4 .

\section{Example I: Analysis of Boeing 720 jet airplanes based on LR}

The first data set consists of number of successive failure for the air conditioning system reported of each member in a fleet of 13 Boeing 720 jet airplanes studied by Tahir et al. (2015). Since the maximum number of successive failure is 603 , the original data were transformed to be in the interval $[0,1]$ using $t=y / \max (y)$. Before progressing further, we have first fitted the GTL distribution, and obtained Kolmogorov-Smirnov distance and the corresponding $p$-value which are 0.0586 and 0.5699 , respectively. Therefore, we can conclude that the GTL distribution is a suitable model for these data sets. It is worth mentionning here that many authors analyzed this data set using well known and some recently proposed distributions with more than three parameters. Studying the properties and the inferential aspects of these distributions based on $d g o s$ is very complicated because of the large numbers of parameters. Therefore, the GTL distribution is a good choice for analyzing such data under dgos. The fitted estimated survival function and P-P plot of the GTL distribution are displayed in Fig. 1. Now, based on the transformed data set, LR values with sample size nine can be generated. These data are displayed in Table 6. The MLEs and Bayes estimates of the parameters $\tau$ and $\delta$ along with their standard error/square root of Bayes risk (SE) based on LR data in Table 6 are obtained and 
Table 5

The MLEs, Bayes estimates, the corresponding SE (within parentheses) and the confidence/credible interval estimates based on LR data

\begin{tabular}{ccc}
\hline Parameter & MLE & Bayes estimates \\
\hline$\tau$ & $331.09(177.45)$ & $330.629(9.876)$ \\
& $(0.000,447.40)$ & $(310.95,349.74)$ \\
$\delta$ & $0.0259(0.0508)$ & $0.0256(0.0034)$ \\
& $(0.000,0.1493)$ & $(0.0189,0.0325)$
\end{tabular}

Table 6

Recovery rate of COVID-19 in Spain

\begin{tabular}{llllllllll}
0.667 & 0.500 & 0.490 & 0.429 & 0.750 & 0.653 & 0.516 & 0.789 & 0.769 & 0.687 \\
0.520 & 0.725 & 0.638 & 0.608 & 0.659 & 0.629 & 0.571 & 0.592 & 0.606 & 0.592 \\
0.592 & 0.559 & 0.595 & 0.616 & 0.646 & 0.672 & 0.684 & 0.685 & 0.695 & 0.707 \\
0.721 & 0.732 & 0.741 & 0.751 & 0.752 & 0.755 & 0.764 & 0.771 & 0.776 & 0.781 \\
0.784 & 0.785 & 0.787 & 0.790 & 0.796 & 0.793 & 0.791 & 0.796 & 0.797 & 0.798 \\
0.801 & 0.804 & 0.829 & 0.832 & 0.835 & 0.837 & 0.839 & 0.846 & 0.849 & 0.852 \\
0.854 & 0.855 & 0.856 & 0.858 & 0.860 & 0.863 & & & & \\
\hline
\end{tabular}
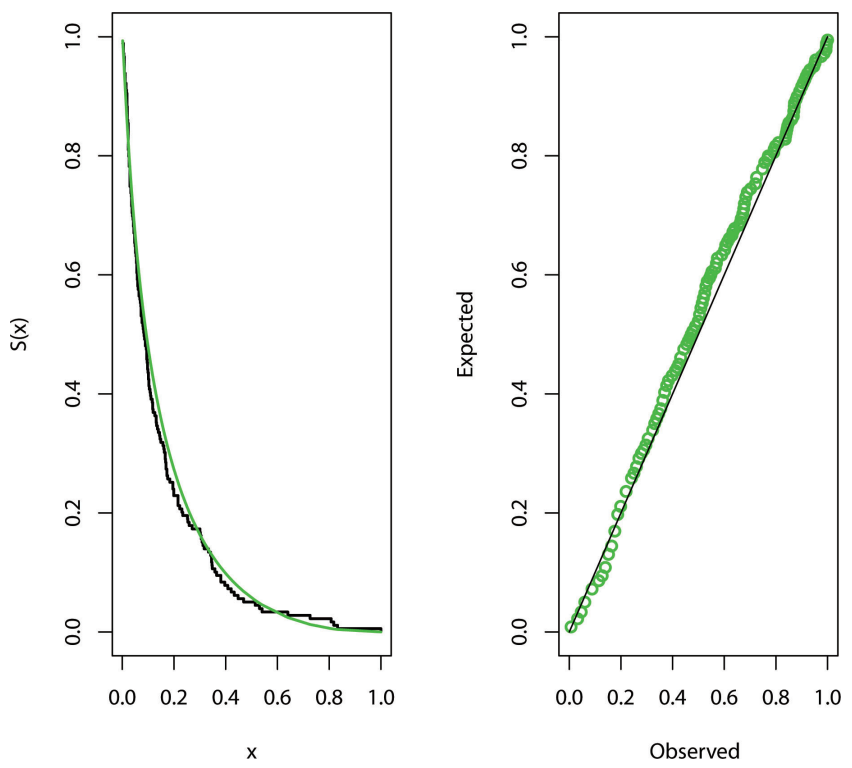

Fig. 1. Estimated SF and P-P plots for real data 1.

displayed in Table 7. The Bayes estimates are obtained based on 12000 MCMC samples with 2000 samples as a burn-in period. Also, the approximate confidence intervals as well as the credible intervals are obtained and presented in Table 5. From Table 5, we can conclude that the Bayes estimates perform better than MLEs based on SE and confidence length.

\section{Example II: Analysis of recovery rate of COVID-19 in Spain based on OS}

The second data set presents the daily recovery rate of COVID-19 in Spain from March 3 to May 7. The data consists of 66 daily recovery rate and available in https:/www.worldometers.info/coronavirus/country/spain/. The data set is presented in Table 6. Since the data consists of the recovery rates of COVID-19 in Spain, which is a unit interval data, one of the choices to model this data set is the beta distribution. It is not easy to analyze the beta distribution under $d g o s$ because its cdf contains an incomplete beta function. In this case, the GTL distribution can be used as a good model with flexible pdf and cdf to analyze this data set as it is measured in the unit interval $(0,1)$. We 
Table 7

The MLEs, Bayes estimates, the corresponding SE (within parentheses) and the confidence/ credible interval estimates based OS data

\begin{tabular}{ccc}
\hline Parameter & MLE & Bayes estimates \\
\hline$\tau$ & $24557.3(164118.8)$ & $24303.47(493.501)$ \\
& $(0.000,346230.21)$ & $(23364.4,25280.7)$ \\
$\delta$ & $0.01720(0.0577)$ & $0.01721(0.00073)$ \\
& $(0.000,0.1303)$ & $(0.01577,0.01866)$ \\
\hline
\end{tabular}
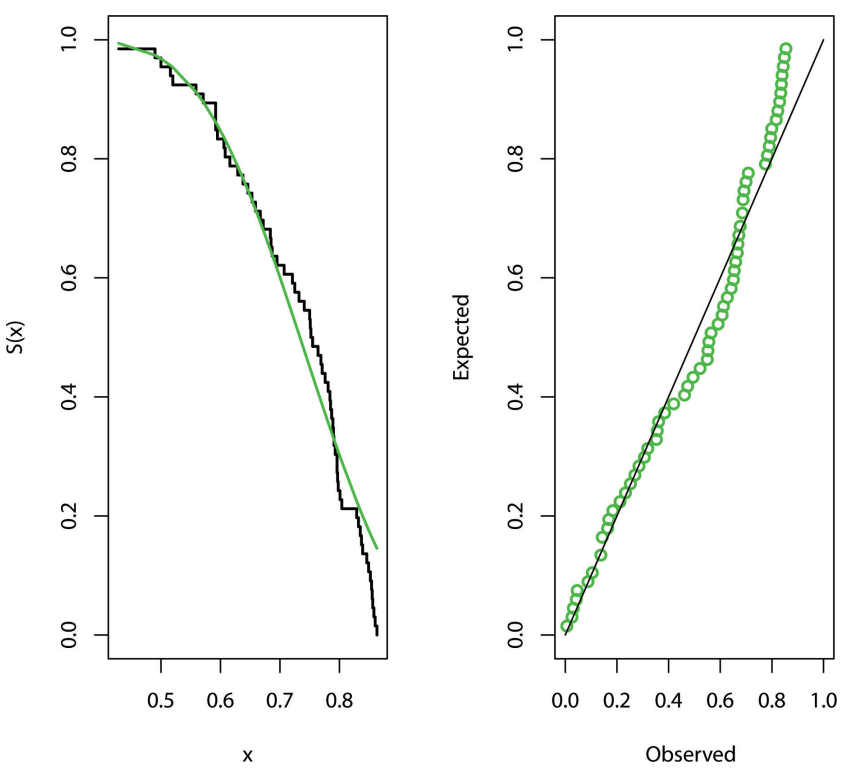

Fig. 2. Estimated SF and P-P plots for real data 2.

first check whether the GTL distribution can be model to these data set. The Kolmogorov-Smirnov distance and the corresponding $p$-value are 0.1456 and 0.1219 , respectively which reveals that the GTL distribution fits the real life data set as well. The fitted estimated survival function and P-P plot of the GTL distribution are displayed in Fig. 2. Based on OS, the MLEs and Bayes estimates and the corresponding SEs of the GTL distribution parameters are presented in Table 7. In addition, the approximate confidence intervals as well as the credible intervals are obtained and displayed in Table 7. From these results, we can conclude that the Bayes estimates perform better than MLEs in terms of minimum SE. Also, the Bayes credible intervals have shorter confidence lengths than the approximate confidence intervals.

\section{Conclusion}

In this paper, first we have obtained the explicit expression for the single and product moments of $d g o s$ from GTL distribution. The results obtained in this paper are more generalized in the sense that it includes the moment of order statistics and lower records from GTL distribution. Further, ML and Bayes methods of estimation are used for estimation of the parameters of the GTL distribution based on order statistics and lower record values. A simulation study is carried out to compare the proposed estimators in terms of RMSE and RAB. In addition, ACIs and HPD credible intervals are compared in terms of their AL and CPs. From simulation and real data analysis, we observe that Bayesian approach is quite satisfactory as compared to non-Bayesian procedure for both OS and LR values. Although many properties of GTL distribution have been discussed recently, it seems that BLUEs/BLUPS of the parameters and prediction of future observations based on ordered data for this distribution have not been investigated yet. The work is in progress and it will be reported later. 


\section{Acknowledgments}

The authors are grateful for the comments and suggestions by the referees and the associate editor. Their comments and suggestions have greatly improved the article.

\section{References}

Ahsanullah, M. (2004). A characterization of the uniform distribution by dual generalized order statistics. Communications in Statistics-Theory and Methods, 33, 2921-2928.

Ahsanullah, M. (2005). On lower generalized order statistics and a characterization of power function distribution. Statistical Methods, 7, 16-28.

Arnold, B. C., \& Press, S. J. (1983). Bayesian inference for Pareto populations. Journal of Econometrics, 21, $287-306$.

Barakat, H. M., \& El-Adll, M. E. (2009). Asymptotic theory of extreme dual generalized order statistics. Stat Probabil Lett, 79, $1252-1259$.

Burkschat, M., Cramer, E., \& Kamps, U. (2003). Dual generalized order statistics. Metron LXI, 13-26.

Chen, M. H., \& Shao, Q. M. (1999). Monte Carlo estimation of Bayesian credible and HPD intervals. Journal of Computational and Graphical Statistics, 8, 69-92.

Cohen, A. C. (1965). Maximum likelihood estimation in the Weibull distribution based on complete and censored samples. Technometrics, 5, $579-588$.

Dey, S., Ali, S., Park, C. (2015). Weighted exponential distribution: Properties and different methods of estimation. J Stat Comput Simul, 85, 3641-3661.

Dey, S., Dey, T., Ali, S., \& Mulekar, M. S. (2016a). Two-parameter Maxwell distribution: Properties and different methods of estimation. J Stat Theory Prac, 10, 291-310.

Dey, S., Singh, S., \& Tripathi, Y. M. (2016b). Estimation and prediction for a progressively censored generalized inverted exponential distribution. Stat Methodol, 32, 185-202.

Genç, A. I. (2013). Estimation of $P(X>Y)$ with ToppâĂŞLeone distribution. J Stat Comput Simul, 83, 326-339.

Ghosh, I., Dey, S., \& Kumar, D. (2019). Bounded M-O extended exponential distribution with applications. Stochastics and Quality Control, 34, 35-51.

Henningsen, A., \& Toomet, O. (2011). 'MaxLik': A package for maximum likelihood estimation in R. Computational Statistics, $26,443-458$.

Jaheen, Z. F., \& Al Harbi, M. M. (2011). Bayesian estimation based on dual generalized order statistics from the exponentiated Weibull model. $J$ Stat Theory Appl, 10, 591-602.

Kamps, U. (1995). A concept of generalized order statistics. B.G. Teubner Stuttgart.

Khan, R. U., Anwar, Z., \& Athar, H. (2008). Recurrence relations for single and product moments of dual generalized order from exponentiatedWeibull distribution. Aligarh J Statist, 28, 37-45.

Khan, R. U., \& Kumar, D. (2010). On moments of generalized order statistics fromexponentiated Pareto distribution and its characterization. Applied Mathematical Sciences (Ruse), 4, 2711-2722.

Khan, R. U., \& Kumar, D. (2011). Expectation identities of lower generalized order statistics from generalized exponential distribution and its characterization. Mathematical Methods of Statistics, 20, 150-157.

Khan, R. U., \& Khan, M. A. (2015). Dual generalized order statistics from family of J-shaped distribution and its characterization. Journal of King Saud University - Science, 27, 285-291.

Khan, M. J. S., \& Iqrar, S. (2019). On moments of dual generalized order statistics from Topp-Leone distribution. Communication in StatisticsTheory and Methods, 48, 479-492.

Kundu, D., \& Howlader, H. (2010). Bayesian inference and prediction of the inverse Weibull distribution for type-II censored data. Comput Stat Data Anal, 54, 1547-1558.

Kundu, D., \& Pradhan, B. (2011). Bayesian analysis of progressively censored competing risks data. Sankhya B, 73, $276-296$.

Kumar, D. (2013a). On moments of lower generalized order statistics from exponentiated Lomax distribution. American Journal of Mathematical and Management Sciences, 32, 238-256.

Kumar, D. (2013b). Relations for marginal and joint moment generating functions of Marshall-Olkin extended logistic distribution based on lower generalized order statistics and characterization. American Journal of Mathematical and Management Sciences, 32, 19-39.

Kumar, D. (2016). Lower generalized order statistics based on inverse Burr distribution. American Journal of Mathematical and Management Sciences, 35, 15-35.

Kumar, D., \& Dey, S. (2017). Relations for moments of generalized order statistics from extended exponential distribution. American Journal of Mathematical and Management Sciences, 36, 378-400.

Kumar, D., Nassar, M., \& Dey, S. (2020). Inference for generalized inverse Lindley distribution based on generalized order statistics. Afrika Matematika, 31, 1207-1235.

Lawless, J. F. (2003). Statistical models and methods for lifetime data. John Wiley and Sons.

Lai, M. T. (2013). Optimum number of minimal repairs for a system under increasing failure rate shock model with cumulative repair-cost limit. International Journal of Reliability and Safety, 7, 95-107.

Li, L. (2016). Bayes estimation of Topp-Leone distribution under symmetric entropy loss function based on lower record values. Science J Appl Math Statist, 4, 284-288.

Mathai, A. M., \& Saxena, R. K. (1973). Generalized hypergeometric functions with applications in statistics and physical science. Lecture Notes in Mathematics, 348, Berlin: Springer-Verlag.

Mbah, A. K., \& Ahsanullah, M. (2007). Some characterization of the power function distribution based on lower generalized order statistics Pakistan J Statist, 23, 139-46. 
Papke, L. E., \& Wooldridge, J. M. (1996). Econometric methods for fractional response variables with an application to 401(k) plan participation rates. J Appl Econ, 11, 619-632.

Pawlas, P., \& Szynal, D. (2001). Recurrence relations for single and product moments of lower generalized order from the inverse Weibull distribution. Demonstratio Mathematica, 34, 353-58.

Plummer, M., Best, N., Cowles, K., \& Vines, K. (2006). CODA: convergence diagnosis and output analysis for MCMC. R news, 6, 7-11.

Rajarshi, S., \& Rajarshi, M. B. (1988). Bathtub distributions: A review. Comm Statist Theory Methods, 17, $2597-2621$.

Shekhawat, K., \& Sharma, V. K. (2020). An extension of J-shaped distribution with application to tissue damage proportions in blood. Sankhya B: The Indian Journal of Statistics. doi: 10.1007/s13571-019-00218-6.

Smithson, M., \& Shou, Y. (2017). CDF-quantile distributions for modelling random variables on the unit interval. British Journal of Mathematical and Statistical Psychology, 70, 412-438.

Tahir, M. H., Hussain, M. A., Cordeiro, G. M., Hamedani, G. G., Mansoor, M., \& Zubair, M. (2015). The Gumbel-Lomax distribution: Properties and applications. Journal of Statistical Theory and Applications, 15, 61-79.

Woosley, R. L., \& Cossman, J. (2007). Drug development and the FDA's critical path initiative. Public Policy, 81, 129-133.

Zghoul, A. A. (2010). Order statistics from a family of J-shaped distributions. Metron, 68, 127-36.

Zghoul, A. A. (2011). Record values from a family of J-shaped distributions. Statistica, 71, 355-65.

\section{Appendix}

Lemma 1. For positive real numbers $p_{1}$ and $p_{2}$, let

$$
\Delta_{1}\left(p_{1}, p_{2}\right)=\int_{0}^{1} y^{p_{1}-1}\left(2-y^{\alpha}\right)^{p_{2}-1} \mathrm{~d} y .
$$

Then

$$
\Delta_{1}\left(p_{1}, p_{2}\right)=2^{p_{2}-1} p_{1}^{-1} 2 F_{1}\left(\frac{p_{1}}{\alpha}, 1-p_{2} ; \frac{p_{1}}{\alpha}+1, \frac{1}{2}\right),
$$

where ${ }_{2} F_{1}\left(b_{1}, b_{2}, b_{3} ; y\right)$ denotes the Gauss hypergeometric function defined by

$$
{ }_{2} F_{1}\left(b_{1}, b_{2}, b_{3} ; y\right)=\sum_{k=0}^{\infty} \frac{\left(b_{1}\right)_{k}\left(b_{2}\right)_{k} y^{k}}{\left(b_{3}\right)_{k} k !},
$$

where $(f)_{p}=f(f+1) \ldots(f+p-1)$ denotes the ascending factorial.

Proof We have

$$
\begin{aligned}
\Delta_{1}\left(p_{1}, p_{2}\right) & =\int_{0}^{1} y^{p_{1}-1}\left(2-y^{\alpha}\right)^{p_{2}-1} \mathrm{~d} y \\
& =\frac{2^{\frac{p_{1}}{\alpha}+p_{2}}-1}{\alpha} \int_{0}^{1} u^{\frac{p_{1}}{\alpha}-1}(1-z)^{p_{2}-1} \mathrm{~d} z \\
& =\frac{2^{\frac{p_{1}}{\alpha}+p_{2}}-1}{\alpha} \operatorname{Bet}\left(\frac{p_{1}}{\alpha}, p_{2}, \frac{1}{2}\right),
\end{aligned}
$$

where $y^{\alpha}=2 z$ and $\operatorname{Bet}\left(a, b, \frac{1}{2}\right)$ be the incomplete beta function defined by $\operatorname{Bet}(a, b, y)=\int_{0}^{y} z^{a-1}(1-z)^{b-1} \mathrm{~d} z$. The result follows by using (Mathai \& Saxena (1973), p. 43). The proof is complete.

Lemma 2. For positive real numbers $p_{1}, p_{2}, q_{1}$ and $q_{2}$, let

$$
\Delta_{2}\left(p_{1}, p_{2}, q_{1}, q_{2}\right)=\int_{0}^{1} \int_{0}^{y} y^{p_{1}}\left(2-y^{\alpha}\right)^{p_{2}} z^{q_{1}}\left(2-z^{\alpha}\right) \mathrm{d} z \mathrm{~d} y
$$

Then

$$
\Delta_{2}\left(p_{1}, p_{2}, q_{1}, q_{2}\right)=\frac{2^{p_{2}+q_{2}}}{\left(q_{1}+1\right)\left(\frac{q_{1}+1}{\alpha}+p_{1}+1\right)} F_{1: 1 ; 0}^{1: 2 ; 1}\left(\begin{array}{l}
\frac{\frac{q_{1}+1}{\alpha}+p_{1}+1: \frac{q_{1}}{\alpha}+1,-q_{2} ;-p_{2}}{\frac{q_{1}+1}{\alpha}+p_{1}+1}+1: \frac{1}{2}+q_{2}+2 ;-\quad ;-
\end{array}\right),
$$

where $F_{l: m ; n}^{p: q ; k}$ denotes the is Kampé de Feriet's Function defined by

$$
F_{l: m ; n}^{p: q ; k}\left(\begin{array}{c}
\left(a_{p}\right):\left(b_{q}\right) ;\left(c_{k}\right) \\
\left(\alpha_{l}\right):\left(\beta_{m}\right) ;\left(\gamma_{n}\right)
\end{array} x, y\right)=\sum_{r=0}^{\infty} \sum_{r=0}^{\infty} \frac{\prod_{j=1}^{p}\left(a_{j}\right)_{r+s} \prod_{j=1}^{q}\left(b_{j}\right)_{r} \prod_{j=1}^{k}\left(c_{j}\right)_{s}}{\prod_{j=1}^{p}\left(\alpha_{j}\right)_{r+s} \prod_{j=1}^{q}\left(\beta_{j}\right)_{r} \prod_{j=1}^{k}\left(\gamma_{j}\right)_{s}} \frac{x^{r}}{r !} \frac{y^{s}}{s !} .
$$




\section{Proof We have}

$$
\begin{aligned}
& \Delta_{2}\left(p_{1}, p_{2}, q_{1}, q_{2}\right)=\int_{0}^{1} y^{p_{1}}\left(2-y^{\alpha}\right)^{p_{2}}\left(\int_{0}^{y} z^{q_{1}}\left(2-z^{\alpha}\right) \mathrm{d} z\right) \mathrm{d} y \\
& =\frac{2^{\frac{q_{1}+1}{\alpha}+q_{2}}}{\alpha} \int_{0}^{1} y^{p_{1}}\left(2-y^{\alpha}\right)^{p_{2}}\left(\int_{0}^{\frac{y}{2}} t^{\frac{q_{1}+1}{\alpha}-1}(1-t)^{q_{2}} \mathrm{~d} t\right) \mathrm{d} y \\
& =\frac{2^{\frac{q_{1}+1}{\alpha}+q_{2}}}{\alpha} \int_{0}^{1} y^{p_{1}}\left(2-y^{\alpha}\right)^{p_{2}} \operatorname{Bet}\left(\frac{q_{1}+1}{\alpha}, q_{2}+1, \frac{y}{2}\right) \mathrm{d} y,
\end{aligned}
$$

where $z^{\alpha}=2 t$. By using the relation between incomplete beta function and Gauss hypergeometric function

$$
\operatorname{Bet}(a, b, y)=\int_{0}^{y} t^{a-1}(1-t)^{b-1} \mathrm{~d} t=a^{-1} y_{2}^{a} F_{1}(a, 1-b, a+1 ; y) .
$$

Now by using Eq. (28), we obtain

$$
\Delta_{2}\left(p_{1}, p_{2}, q_{1}, q_{2}\right)=\frac{2^{q_{2}}}{q_{1}+1} \int_{0}^{1} y^{p_{1}+\frac{q_{1}+1}{\alpha}}\left(2-y^{\alpha}\right)^{p_{2}} 2 F_{1}\left(\frac{q_{1}+1}{\alpha},-q_{2} ; \frac{q_{1}+1}{\alpha}+1, \frac{y}{2}\right) \mathrm{d} y .
$$

In view of Eq. (25), $\Delta_{2}\left(p_{1}, p_{2}, q_{1}, q_{2}\right)$ can be rewritten as

$$
\begin{aligned}
& \Delta_{2}\left(p_{1}, p_{2}, q_{1}, q_{2}\right)=\frac{2^{q_{2}}}{q_{1}+1} \sum_{i=0}^{\infty} \frac{\left(\frac{q_{1}+1}{\alpha}\right)_{i}\left(-q_{2}\right)_{i}}{\left(\frac{q_{1}+1}{\alpha}\right)_{i} i} 2^{-i} \int_{0}^{1} y^{p_{1}+\frac{q_{1}+1}{\alpha}+i}\left(2-y^{\alpha}\right)^{p_{2}} \mathrm{~d} y \\
& =\sum_{i=0}^{\infty} \frac{\left(\frac{q_{1}+1}{\alpha}\right)_{i}\left(-q_{2}\right)_{i}}{\left(\frac{q_{1}+1}{\alpha}\right)_{i} i !\left(q_{1}+1\right)} 2^{-i} \frac{1}{\alpha} 2^{p_{2}+q_{2}+\frac{q_{1}+1}{\alpha}+p_{1}+i+1} \\
& \times \int_{0}^{\frac{1}{2}} v^{\frac{\frac{q_{1}+1}{\alpha}+p_{1}+i+1}{\alpha}-1}(1-v)^{p_{2}} \mathrm{~d} v
\end{aligned}
$$

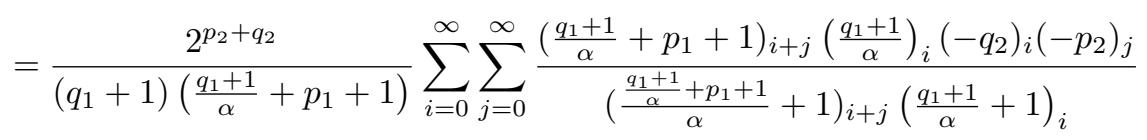

$$
\begin{aligned}
& \times \frac{2^{-i} 2^{-j}}{\left(\frac{q_{1}+1}{\alpha}+p_{1}+i+1\right)} \frac{1}{i ! j !},
\end{aligned}
$$

where $y^{\alpha}=2 v$. The result follows by using Eq. (27). The proof is complete. 